\title{
Public Key Encryption with Keyword Search
}

\author{
Dan Boneh $^{1 \star}$, Giovanni Di Crescenzo ${ }^{2}$, Rafail Ostrovsky ${ }^{3 \star \star}$, and \\ Giuseppe Persiano ${ }^{4 \star \star \star}$ \\ 1 Stanford University \\ dabo@cs.stanford.edu \\ 2 Telcordia \\ giovanni@research.telcordia.com \\ 3 UCLA \\ rafail@cs.ucla.edu \\ 4 Università di Salerno \\ giuper@dia.unisa.it
}

\begin{abstract}
We study the problem of searching on data that is encrypted using a public key system. Consider user Bob who sends email to user Alice encrypted under Alice's public key. An email gateway wants to test whether the email contains the keyword "urgent" so that it could route the email accordingly. Alice, on the other hand does not wish to give the gateway the ability to decrypt all her messages. We define and construct a mechanism that enables Alice to provide a key to the gateway that enables the gateway to test whether the word "urgent" is a keyword in the email without learning anything else about the email. We refer to this mechanism as Public Key Encryption with keyword Search. As another example, consider a mail server that stores various messages publicly encrypted for Alice by others. Using our mechanism Alice can send the mail server a key that will enable the server to identify all messages containing some specific keyword, but learn nothing else. We define the concept of public key encryption with keyword search and give several constructions.
\end{abstract}

\section{Introduction}

Suppose user Alice wishes to read her email on a number of devices: laptop, desktop, pager, etc. Alice's mail gateway is supposed to route email to the appropriate device based on the keywords in the email. For example, when Bob sends email with the keyword "urgent" the mail is routed to Alice's pager. When Bob sends email with the keyword "lunch" the mail is routed to Alice's desktop for reading later. One expects each email to contain a small number of keywords. For example, all words on the subject line as well as the sender's email address

\footnotetext{
* Supported by NSF and the Packard foundation.

** Partially supported by a gift from Teradata. Preliminary work done while visiting Stanford and while at Telcordia.

*** Part of this work done while visiting DIMACS. Work supported by NoE ECRYPT. 
could be used as keywords. The mobile people project 24] provides this email processing capability.

Now, suppose Bob sends encrypted email to Alice using Alice's public key. Both the contents of the email and the keywords are encrypted. In this case the mail gateway cannot see the keywords and hence cannot make routing decisions. As a result, the mobile people project is unable to process secure email without violating user privacy. Our goal is to enable Alice to give the gateway the ability to test whether "urgent" is a keyword in the email, but the gateway should learn nothing else about the email. More generally, Alice should be able to specify a few keywords that the mail gateway can search for, but learn nothing else about incoming mail. We give precise definitions in section 2

To do so, Bob encrypts his email using a standard public key system. He then appends to the resulting ciphertext a Public-Key Encryption with keyword Search (PEKS) of each keyword. To send a message $M$ with keywords $W_{1}, \ldots, W_{m}$ Bob sends

$$
E_{A_{p u b}}(M)\left\|\operatorname{PEKS}\left(A_{p u b}, W_{1}\right)\right\| \cdots \| \operatorname{PEKS}\left(A_{p u b}, W_{m}\right)
$$

Where $A_{p u b}$ is Alice's public key. The point of this form of encryption is that Alice can give the gateway a certain trapdoor $T_{W}$ that enables the gateway to test whether one of the keywords associated with the message is equal to the word $W$ of Alice's choice. Given $\operatorname{PEKS}\left(A_{p u b}, W^{\prime}\right)$ and $T_{W}$ the gateway can test whether $W=W^{\prime}$. If $W \neq W^{\prime}$ the gateway learns nothing more about $W^{\prime}$. Note that Alice and Bob do not communicate in this entire process. Bob generates the searchable encryption for $W^{\prime}$ just given Alice's public key.

In some cases, it is instructive to view the email gateway as an IMAP or POP email server. The server stores many emails and each email contains a small number of keywords. As before, all these emails are created by various people sending mail to Alice encrypted using her public key. We want to enable Alice to ask queries of the form: do any of the messages on the server contain the keyword "urgent"? Alice would do this by giving the server a trapdoor $T_{W}$, thus enabling the server to retrieve emails containing the keyword $W$. The server learns nothing else about the emails.

Related work. A related issue deals with privacy of database data. There are two different scenarios: public databases and private databases, and the solutions for each are different.

Private databases: In this settings a user wishes to upload its private data to a remote database and wishes to keep the data private from the remote database administrator. Later, the user must be able to retrieve from the remote database all records that contain a particular keyword. Solutions to this problem were presented in the early 1990's by Ostrovsky [26] and Ostrovsky and Goldreich [17] and more recently by Song at al. 28. The solution of Song. at al 28, requires very little communication between the user and the database (proportional to the security parameter) and only one round of interaction. The database performs work that is linear in its size per query. The solution of 2617] requires poly-logarithmic rounds (in the size of the database) between the user and the 
database, but allows the database to do only poly-logarithmic work per query. An additional privacy requirement that might be appealing in some scenarios is to hide from the database administrator any information regarding the access pattern, i.e. if some item was retrieved more then once, some item was not retrieved at all, etc. The work of [26 17] achieves this property as well, with the same poly-logarithmic cost5 per query both for the database-user interaction and the actual database work. We stress that both the constructions of 2617 and the more recent work of [10[28]16] apply only to the private-key setting for users who own their data and wish to upload it to a third-party database that they do not trust.

Public Databases Here the database data is public (such as stock quotes) but the user is unaware of it and wishes to retrieve some data-item or search for some data-item, without revealing to the database administrator which item it is. The naive solution is that the user can download the entire database. Public Information Retrieval (PIR) protocols allow user to retrieve data from a public database with far smaller communication then just downloading the entire database. PIR was first shown to be possible only in the setting where there are many copies of the same database and none of the copies can talk to each other [5. PIR was shown to be possible for a single database by Kushilevitz and Ostrovsky 22] (using homomorphic encryption scheme of [19]). The communication complexity of [22] solution (i.e. the number of bits transmitted between the user and the database) is $O\left(n^{\epsilon}\right)$, where $n$ is the size of the database and $\epsilon>0$. This was reduced to poly-logarithmic overhead by Cachin, Micali, and Stadler [4]. As pointed out in [22], the model of PIR can be extended to one-out-of- $n$ Oblivious Transfer and keyword searching on public data, and received a lot of additional attention in the literature (see, for example, [22]8|20|9|23|25|27]. We stress though that in all these settings the database is public, and the user is trying to retrieve or find certain items without revealing to the database administrator what it is searching for. In the setting of a single public database, it can be shown that the database must always perform work which is at least linear in the size of the database.

Our problem does not fit either of the two models mentioned above. Unlike the private-key setting, data collected by the mail-server is from third parties, and can not be "organized" by the user in any convenient way. Unlike the publicly available database, the data is not public, and hence the PIR solutions do not apply.

We point out that in practical applications, due to the computation cost of public key encryption, our constructions are applicable to searching on a small number of keywords rather than an entire file. Recently, Waters et al. 30 showed that public key encryption with keyword search can be used to build an encrypted and searchable audit log. Other methods for searching on encrypted data are described in [16]12.

\footnotetext{
${ }^{5}$ The poly-logarithmic construction of 26 17] requires large constants, which makes it impractical; however their basic $O(\sqrt{n})$ solution was recently shown to be applicable for some practical applications [10].
} 


\section{Public Key Encryption with Searching: Definitions}

Throughout the paper we use the term negligible function to refer to a function $f: \mathbb{R} \rightarrow[0,1]$ where $f(s)<1 / g(s)$ for any polynomial $g$ and sufficiently large $s$.

We start by precisely defining what is a secure Public Key Encryption with keyword Search (PEKS) scheme. Here "public-key" refers to the fact that ciphertexts are created by various people using Alice's public key. Suppose user Bob is about to send an encrypted email to Alice with keywords $W_{1}, \ldots, W_{k}$ (e.g., words in the subject line and the sender's address could be used as keywords, so that $k$ is relatively small). Bob sends the following message:

$$
\left[E_{A_{p u b}}[m s g], \operatorname{PEKS}\left(A_{p u b}, W_{1}\right), \ldots, \operatorname{PEKS}\left(A_{p u b}, W_{k}\right)\right]
$$

where $A_{p u b}$ is Alice's public key, $m s g$ is the email body, and PEKS is an algorithm with properties discussed below. The PEKS values do not reveal any information about the message, but enable searching for specific keywords. For the rest of the paper, we use as our sample application a mail server that stores all incoming email.

Our goal is to enable Alice to send a short secret key $T_{W}$ to the mail server that will enable the server to locate all messages containing the keyword $W$, but learn nothing else. Alice produces this trapdoor $T_{W}$ using her private key. The server simply sends the relevant emails back to Alice. We call such a system non-interactive public key encryption with keyword search, or as a shorthand "searchable public-key encryption".

Definition 1. A non-interactive public key encryption with keyword search (we sometimes abbreviate it as "searchable encryption") scheme consists of the following polynomial time randomized algorithms:

1. KeyGen(s): Takes a security parameter, $s$, and generates a public/private key pair $A_{\text {pub }}, A_{\text {priv }}$.

2. $\operatorname{PEKS}\left(A_{p u b}, W\right)$ : for a public key $A_{p u b}$ and a word $W$, produces a searchable encryption of $W$.

3. Trapdoor $\left(A_{\text {priv }}, W\right)$ : given Alice's private key and a word $W$ produces a trapdoor $T_{W}$.

4. Test $\left(A_{\text {pub }}, S, T_{W}\right)$ : given Alice's public key, a searchable encryption $S=$ $\operatorname{PEKS}\left(A_{\text {pub }}, W^{\prime}\right)$, and a trapdoor $T_{W}=\operatorname{Trapdoor}\left(A_{\text {priv }}, W\right)$, outputs 'yes' if $W=W^{\prime}$ and 'no' otherwise.

Alice runs the KeyGen algorithm to generate her public/private key pair. She uses Trapdoor to generate trapdoors $T_{W}$ for any keywords $W$ that she wants the mail server or mail gateway to search for. The mail server uses the given trapdoors as input to the Test() algorithm to determine whether a given email contains one of the keywords $W$ specified by Alice.

Next, we define security for a PEKS in the sense of semantic-security. We need to ensure that an $\operatorname{PEKS}\left(A_{\text {pub }}, W\right)$ does not reveal any information about $W$ unless $T_{W}$ is available. We define security against an active attacker who is able to obtain trapdoors $T_{W}$ for any $W$ of his choice. Even under such attack 
the attacker should not be able to distinguish an encryption of a keyword $W_{0}$ from an encryption of a keyword $W_{1}$ for which he did not obtain the trapdoor. Formally, we define security against an active attacker $\mathcal{A}$ using the following game between a challenger and the attacker (the security parameter $s$ is given to both players as input).

PEKS Security game:

1. The challenger runs the $\operatorname{KeyGen}(s)$ algorithm to generate $A_{\text {pub }}$ and $A_{\text {priv }}$. It gives $A_{p u b}$ to the attacker.

2. The attacker can adaptively ask the challenger for the trapdoor $T_{W}$ for any keyword $W \in\{0,1\}^{*}$ of his choice.

3. At some point, the attacker $\mathcal{A}$ sends the challenger two words $W_{0}, W_{1}$ on which it wishes to be challenged. The only restriction is that the attacker did not previously ask for the trapdoors $T_{W_{0}}$ or $T_{W_{1}}$. The challenger picks a random $b \in\{0,1\}$ and gives the attacker $C=\operatorname{PEKS}\left(A_{p u b}, W_{b}\right)$. We refer to $C$ as the challenge PEKS.

4. The attacker can continue to ask for trapdoors $T_{W}$ for any keyword $W$ of his choice as long as $W \neq W_{0}, W_{1}$.

5. Eventually, the attacker $\mathcal{A}$ outputs $b^{\prime} \in\{0,1\}$ and wins the game if $b=b^{\prime}$.

In other words, the attacker wins the game if he can correctly guess whether he was given the PEKS for $W_{0}$ or $W_{1}$. We define $\mathcal{A}$ 's advantage in breaking the PEKS as

$$
\operatorname{Adv}_{\mathcal{A}}(s)=\left|\operatorname{Pr}\left[b=b^{\prime}\right]-\frac{1}{2}\right|
$$

Definition 2. We say that a PEKS is semantically secure against an adaptive chosen keyword attack if for any polynomial time attacker $\mathcal{A}$ we have that $\operatorname{Adv}_{\mathcal{A}}(s)$ is a negligible function.

Chosen Ciphertext Security. We note that Definition2 ensures that the construction given in Eq. (1I) is semantically secure whenever the public key encryption system $E_{A_{p u b}}$ is semantically secure. However, as is, the construction is not chosen ciphertext secure. Indeed, a chosen ciphertext attacker can break semantic security by reordering the keywords in Eq. (1) and submitting the resulting ciphertext for decryption. A standard technique can make this construction chosen ciphertext secure using the methods of [7]. We defer this to the full version of the paper.

\subsection{PEKS Implies Identity Based Encryption}

Public key encryption with keyword search is related to Identity Based Encryption (IBE) 292]. Constructing a secure PEKS appears to be a harder problem than constructing an IBE. Indeed, the following lemma shows that PEKS implies Identity Based Encryption. The converse is probably false. Security notions for IBE, and in particular chosen ciphertext secure IBE (IND-ID-CCA), are defined in [2]. 
Lemma 1. A non-interactive searchable encryption scheme (PEKS) that is semantically secure against an adaptive chosen keyword attack gives rise to a chosen ciphertext secure IBE system (IND-ID-CCA).

Proof sketch: Given a PEKS (KeyGen, PEKS, Trapdoor, Test) the IBE system is as follows:

1. Setup: Run the PEKS KeyGen algorithm to generate $A_{\text {pub }} / A_{\text {priv }}$. The IBE system parameters are $A_{\text {pub }}$. The master-key is $A_{\text {priv }}$.

2. KeyGen: The IBE private key associated with a public key $X \in\{0,1\}^{*}$ is

$$
d_{X}=\left[\operatorname{Trapdoor}\left(A_{\text {priv }}, X \| 0\right), \operatorname{Trapdoor}\left(A_{\text {priv }}, X \| 1\right)\right],
$$

where $\|$ denotes concatenation.

3. Encrypt: Encrypt a bit $b \in\{0,1\}$ using a public key $X \in\{0,1\}^{*}$ as: $C T=\operatorname{PEKS}\left(A_{p u b}, X \| b\right)$.

4. Decrypt: To decrypt $C T=\operatorname{PEKS}\left(A_{\text {pub }}, X \| b\right)$ using the private key $d_{X}=$ $\left(d_{0}, d_{1}\right)$ output ' 0 ' if Test $\left(A_{p u b}, C T, d_{0}\right)=$ 'yes' and

output ' 1 ' if Test $\left(A_{\text {pub }}, C T, d_{1}\right)=$ 'yes'

One can show that the resulting system is IND-ID-CCA assuming the PEKS is semantically secure against an adaptive chosen message attack.

This shows that building non-interactive public-key searchable encryption is at least as hard as building an IBE system. One might be tempted to prove the converse (i.e., IBE implies PEKS) by defining

$$
\operatorname{PEKS}\left(A_{p u b}, W\right)=E_{W}\left[0^{k}\right]
$$

i.e. encrypt a string of $k$ zeros with the IBE public key $W \in\{0,1\}^{*}$. The Test algorithm attempts to decrypt $E_{W}[0]$ and checks that the resulting plaintext is $0^{k}$. Unfortunately, this does not necessarily give a secure searchable encryption scheme. The problem is that the ciphertext $C T$ could expose the public key $(W)$ used to create $C T$. Generally, an encryption scheme need not hide the public key that was used to create a given ciphertext. But this property is essential for the PEKS construction given in (2). We note that public key privacy was previously studied by Bellare et al. [1].

Generally, it appears that constructing a searchable public-key encryption is a harder problem than constructing an IBE scheme. Nevertheless, our first PEKS construction is based on a recent construction for an IBE system. We are able to prove security by exploiting extra properties of this system.

\section{Constructions}

We give two constructions for public-key searchable encryption: (1) an efficient system based on a variant of the Decision Diffie-Hellman assumption (assuming a random oracle) and (2) a limited system based on general trapdoor permutations (without assuming the random oracle), but less efficient. 


\subsection{Construction Using Bilinear Maps}

Our first construction is based on a variant of the Computational Diffie-Hellman problem. Boneh and Franklin 2 recently used bilinear maps on elliptic curves to build an efficient IBE system. Abstractly, they use two groups $G_{1}, G_{2}$ of prime order $p$ and a bilinear map $e: G_{1} \times G_{1} \rightarrow G_{2}$ between them. The map satisfies the following properties:

1. Computable: given $g, h \in G_{1}$ there is a polynomial time algorithms to compute $e(g, h) \in G_{2}$.

2. Bilinear: for any integers $x, y \in[1, p]$ we have $e\left(g^{x}, g^{y}\right)=e(g, g)^{x y}$

3. Non-degenerate: if $g$ is a generator of $G_{1}$ then $e(g, g)$ is a generator of $G_{2}$.

The size of $G_{1}, G_{2}$ is determined by the security parameter.

We build a non-interactive searchable encryption scheme from such a bilinear map. The construction is based on 2]. We will need hash functions $H_{1}:\{0,1\}^{*} \rightarrow$ $G_{1}$ and $H_{2}: G_{2} \rightarrow\{0,1\}^{\log p}$. Our PEKS works as follows:

- KeyGen: The input security parameter determines the size, $p$, of the groups $G_{1}$ and $G_{2}$. The algorithm picks a random $\alpha \in \mathbb{Z}_{p}^{*}$ and a generator $g$ of $G_{1}$. It outputs $A_{\text {pub }}=\left[g, h=g^{\alpha}\right]$ and $A_{\text {priv }}=\alpha$.

- PEKS $\left(A_{\text {pub }}, W\right)$ : First compute $t=e\left(H_{1}(W), h^{r}\right) \in G_{2}$ for a random $r \in \mathbb{Z}_{p}^{*}$. Output $\operatorname{PEKS}\left(A_{\text {pub }}, W\right)=\left[g^{r}, H_{2}(t)\right]$.

- Trapdoor $\left(A_{\text {priv }}, W\right)$ : output $T_{W}=H_{1}(W)^{\alpha} \in G_{1}$.

- Test $\left(A_{p u b}, S, T_{W}\right)$ : let $S=[A, B]$. Test if $H_{2}\left(e\left(T_{W}, A\right)\right)=B$. If so, output 'yes'; if not, output 'no'.

We prove that this system is a non-interactive searchable encryption scheme semantically secure against a chosen keyword attack in the random oracle model. The proof of security relies on the difficulty of the Bilinear Diffie-Hellman problem $(\mathrm{BDH})[2 \mid 21$.

Bilinear Diffie-Hellman Problem $(B D H)$ : Fix a generator $g$ of $G_{1}$. The BDH problem is as follows: given $g, g^{a}, g^{b}, g^{c} \in G_{1}$ as input, compute $e(g, g)^{a b c} \in$ $G_{2}$. We say that BDH is intractable if all polynomial time algorithms have a negligible advantage in solving $\mathrm{BDH}$.

We note that the Boneh-Franklin IBE system [2] relies on the same intractability assumption for security. The security of our PEKS is proved in the following theorem. The proof is set in the random oracle model. Indeed, it is currently an open problem to build a secure IBE, and hence a PEKS, without the random oracle model.

Theorem 1. The non-interactive searchable encryption scheme (PEKS) above is semantically secure against a chosen keyword attack in the random oracle model assuming $B D H$ is intractable.

Proof : Suppose $\mathcal{A}$ is an attack algorithm that has advantage $\epsilon$ in breaking the PEKS. Suppose $\mathcal{A}$ makes at most $q_{\mathrm{H}_{2}}$ hash function queries to $H_{2}$ and at 
most $q_{T}$ trapdoor queries (we assume $q_{T}$ and $q_{H_{2}}$ are positive). We construct an algorithm $\mathcal{B}$ that solves the $\mathrm{BDH}$ problem with probability at least $\epsilon^{\prime}=$ $\epsilon /\left(e q_{T} q_{H_{2}}\right)$, where $e$ is the base of the natural logarithm. Algorithm $\mathcal{B}$ 's running time is approximately the same as $\mathcal{A}$ 's. Hence, if the BDH assumption holds in $G_{1}$ then $\epsilon^{\prime}$ is a negligible function and consequently $\epsilon$ must be a negligible function in the security parameter.

Let $g$ be a generator of $G_{1}$. Algorithm $\mathcal{B}$ is given $g, u_{1}=g^{\alpha}, u_{2}=g^{\beta}, u_{3}=$ $g^{\gamma} \in G_{1}$. Its goal is to output $v=e(g, g)^{\alpha \beta \gamma} \in G_{2}$. Algorithm $\mathcal{B}$ simulates the challenger and interacts with forger $\mathcal{A}$ as follows:

KeyGen. Algorithm $\mathcal{B}$ starts by giving $\mathcal{A}$ the public key $A_{\text {pub }}=\left[g, u_{1}\right]$.

$H_{1}, H_{2}$-queries. At any time algorithm $\mathcal{A}$ can query the random oracles $H_{1}$ or $H_{2}$. To respond to $H_{1}$ queries algorithm $\mathcal{B}$ maintains a list of tuples $\left\langle W_{j}, h_{j}, a_{j}, c_{j}\right\rangle$ called the $H_{1}$-list. The list is initially empty. When $\mathcal{A}$ queries the random oracle $H_{1}$ at a point $W_{i} \in\{0,1\}^{*}$, algorithm $\mathcal{B}$ responds as follows:

1. If the query $W_{i}$ already appears on the $H_{1}$-list in a tuple $\left\langle W_{i}, h_{i}, a_{i}, c_{i}\right\rangle$ then algorithm $\mathcal{B}$ responds with $H_{1}\left(W_{i}\right)=h_{i} \in G_{1}$.

2. Otherwise, $\mathcal{B}$ generates a random coin $c_{i} \in\{0,1\}$ so that $\operatorname{Pr}\left[c_{i}=0\right]=$ $1 /\left(q_{T}+1\right)$.

3. Algorithm $\mathcal{B}$ picks a random $a_{i} \in \mathbb{Z}_{p}$.

If $c_{i}=0, \mathcal{B}$ computes $h_{i} \leftarrow u_{2} \cdot g^{a_{i}} \in G_{1}$.

If $c_{i}=1, \mathcal{B}$ computes $h_{i} \leftarrow g^{a_{i}} \in G_{1}$.

4. Algorithm $\mathcal{B}$ adds the tuple $\left\langle W_{i}, h_{i}, a_{i}, c_{i}\right\rangle$ to the $H_{1}$-list and responds to $\mathcal{A}$ by setting $H_{1}\left(W_{i}\right)=h_{i}$. Note that either way $h_{i}$ is uniform in $G_{1}$ and is independent of $\mathcal{A}$ 's current view as required.

Similarly, at any time $\mathcal{A}$ can issue a query to $H_{2}$. Algorithm $\mathcal{B}$ responds to a query for $H_{2}(t)$ by picking a new random value $V \in\{0,1\}^{\log p}$ for each new $t$ and setting $H_{2}(t)=V$. In addition, $\mathcal{B}$ keeps track of all $H_{2}$ queries by adding the pair $(t, V)$ to an $\mathrm{H}_{2}$-list. The $\mathrm{H}_{2}$-list is initially empty.

Trapdoor queries. When $\mathcal{A}$ issues a query for the trapdoor corresponding to the word $W_{i}$ algorithm $\mathcal{B}$ responds as follows:

1. Algorithm $\mathcal{B}$ runs the above algorithm for responding to $H_{1}$-queries to obtain an $h_{i} \in G_{1}$ such that $H_{1}\left(W_{i}\right)=h_{i}$. Let $\left\langle W_{i}, h_{i}, a_{i}, c_{i}\right\rangle$ be the corresponding tuple on the $H_{1}$-list. If $c_{i}=0$ then $\mathcal{B}$ reports failure and terminates.

2. Otherwise, we know $c_{i}=1$ and hence $h_{i}=g^{a_{i}} \in G_{1}$. Define $T_{i}=u_{1}^{a_{i}}$. Observe that $T_{i}=H\left(W_{i}\right)^{\alpha}$ and therefore $T_{i}$ is the correct trapdoor for the keyword $W_{i}$ under the public key $A_{p u b}=\left[g, u_{1}\right]$. Algorithm $\mathcal{B}$ gives $T_{i}$ to algorithm $\mathcal{A}$.

Challenge. Eventually algorithm $\mathcal{A}$ produces a pair of keywords $W_{0}$ and $W_{1}$ that it wishes to be challenged on. Algorithm $\mathcal{B}$ generates the challenge PEKS as follows:

1. Algorithm $\mathcal{B}$ runs the above algorithm for responding to $H_{1}$-queries twice to obtain a $h_{0}, h_{1} \in G_{1}$ such that $H_{1}\left(W_{0}\right)=h_{0}$ and $H_{1}\left(W_{1}\right)=h_{1}$. For $i=0,1$ let $\left\langle W_{i}, h_{i}, a_{i}, c_{i}\right\rangle$ be the corresponding tuples on the $H_{1}$-list. If both $c_{0}=1$ and $c_{1}=1$ then $\mathcal{B}$ reports failure and terminates. 
2. We know that at least one of $c_{0}, c_{1}$ is equal to 0 . Algorithm $\mathcal{B}$ randomly picks a $b \in\{0,1\}$ such that $c_{b}=0$ (if only one $c_{b}$ is equal to 0 then no randomness is needed since there is only one choice).

3. Algorithm $\mathcal{B}$ responds with the challenge PEKS $C=\left[u_{3}, J\right]$ for a random $J \in\{0,1\}^{\log p}$.

Note that this challenge implicitly defines $H_{2}\left(e\left(H_{1}\left(W_{b}\right), u_{1}^{\gamma}\right)\right)=J$. In other words,

$$
J=H_{2}\left(e\left(H_{1}\left(W_{b}\right), u_{1}^{\gamma}\right)\right)=H_{2}\left(e\left(u_{2} g^{a_{b}}, g^{\alpha \gamma}\right)\right)=H_{2}\left(e(g, g)^{\alpha \gamma\left(\beta+a_{b}\right)}\right)
$$

With this definition, $C$ is a valid PEKS for $W_{b}$ as required.

More trapdoor queries. $\mathcal{A}$ can continue to issue trapdoor queries for keywords $W_{i}$ where the only restriction is that $W_{i} \neq W_{0}, W_{1}$. Algorithm $\mathcal{B}$ responds to these queries as before.

Output. Eventually, $\mathcal{A}$ outputs its guess $b^{\prime} \in\{0,1\}$ indicating whether the challenge $C$ is the result of $\operatorname{PEKS}\left(A_{\text {pub }}, W_{0}\right)$ or $\operatorname{PEKS}\left(A_{p u b}, W_{1}\right)$. At this point, algorithm $\mathcal{B}$ picks a random pair $(t, V)$ from the $H_{2}$-list and outputs $t / e\left(u_{1}, u_{3}\right)^{a_{b}}$ as its guess for $e(g, g)^{\alpha \beta \gamma}$, where $a_{b}$ is the value used in the Challenge step. The reason this works is that, as we will show, $\mathcal{A}$ must have issued a query for either $H_{2}\left(e\left(H_{1}\left(W_{0}\right), u_{1}^{\gamma}\right)\right)$ or $H_{2}\left(e\left(H_{1}\left(W_{1}\right), u_{1}^{\gamma}\right)\right)$. Therefore, with probability $1 / 2$ the $H_{2}$-list contains a pair whose left hand side is $t=e\left(H_{1}\left(W_{b}\right), u_{1}^{\gamma}\right)=e(g, g)^{\alpha \gamma\left(\beta+a_{b}\right)}$. If $\mathcal{B}$ picks this pair $(t, V)$ from the $H_{2}$-list then $t / e\left(u_{1}, u_{3}\right)^{a_{b}}=e(g, g)^{\alpha \beta \gamma}$ as required.

This completes the description of algorithm $\mathcal{B}$. It remains to show that $\mathcal{B}$ correctly outputs $e(g, g)^{\alpha \beta \gamma}$ with probability at least $\epsilon^{\prime}$. To do so, we first analyze the probability that $\mathcal{B}$ does not abort during the simulation. We define two events:

$\mathcal{E}_{1}: \mathcal{B}$ does not abort as a result of any of $\mathcal{A}$ 's trapdoor queries.

$\mathcal{E}_{2}: \mathcal{B}$ does not abort during the challenge phase.

We first argue as in [6] that both events $\mathcal{E}_{1}$ and $\mathcal{E}_{2}$ occur with sufficiently high probability.

Claim 1: The probability that algorithm $\mathcal{B}$ does not abort as a result of $\mathcal{A}$ 's trapdoor queries is at least $1 / e$. Hence, $\operatorname{Pr}\left[\mathcal{E}_{1}\right] \geq 1 / e$.

Proof. Without loss of generality we assume that $\mathcal{A}$ does not ask for the trapdoor of the same keyword twice. The probability that a trapdoor query causes $\mathcal{B}$ to abort is $1 /\left(q_{T}+1\right)$. To see this, let $W_{i}$ be $\mathcal{A}$ 's $i$ 'th trapdoor query and let $\left\langle W_{i}, h_{i}, a_{i}, c_{i}\right\rangle$ be the corresponding tuple on the $H_{1}$-list. Prior to issuing the query, the bit $c_{i}$ is independent of $\mathcal{A}$ 's view - the only value that could be given to $\mathcal{A}$ that depends on $c_{i}$ is $H\left(W_{i}\right)$, but the distribution on $H\left(W_{i}\right)$ is the same whether $c_{i}=0$ or $c_{i}=1$. Therefore, the probability that this query causes $\mathcal{B}$ to abort is at most $1 /\left(q_{T}+1\right)$. Since $\mathcal{A}$ makes at most $q_{T}$ trapdoor queries the probability that $\mathcal{B}$ does not abort as a result of all trapdoor queries is at least $\left(1-1 /\left(q_{T}+1\right)\right)^{q_{T}} \geq 1 / e$.

Claim 2: The probability that algorithm $\mathcal{B}$ does not abort during the challenge phase is at least $1 / q_{T}$. Hence, $\operatorname{Pr}\left[\mathcal{E}_{2}\right] \geq 1 / q_{T}$. 
Proof. Algorithm $\mathcal{B}$ will abort during the challenge phase if $\mathcal{A}$ is able to produce $W_{0}, W_{1}$ with the following property: $c_{0}=c_{1}=1$ where for $i=0,1$ the tuple $\left\langle W_{i}, h_{i}, a_{i}, c_{i}\right\rangle$ is the tuple on the $H_{1}$-list corresponding to $W_{i}$. Since $\mathcal{A}$ has not queried for the trapdoor for $W_{0}, W_{1}$ we have that both $c_{0}, c_{1}$ are independent of $\mathcal{A}$ 's current view. Therefore, since $\operatorname{Pr}\left[c_{i}=0\right]=1 /\left(q_{T}+1\right)$ for $i=0,1$, and the two values are independent of one another, we have that $\operatorname{Pr}\left[c_{0}=c_{1}=1\right]=$ $\left(1-1 /\left(q_{T}+1\right)\right)^{2} \leq 1-1 / q_{T}$. Hence, the probability that $\mathcal{B}$ does not abort is at least $1 / q_{T}$.

Observe that since $\mathcal{A}$ can never issue a trapdoor query for the challenge keywords $W_{0}, W_{1}$ the two events $\mathcal{E}_{1}$ and $\mathcal{E}_{2}$ are independent. Therefore, $\operatorname{Pr}\left[\mathcal{E}_{1} \wedge\right.$ $\left.\mathcal{E}_{2}\right] \geq 1 /\left(e q_{T}\right)$.

To complete the proof of Theorem 1 it remains to show that $\mathcal{B}$ outputs the solution to the given $\mathrm{BDH}$ instance with probability at least $\epsilon / q_{H_{2}}$. To do we show that during the simulation $\mathcal{A}$ issues a query for $H_{2}\left(e\left(H_{1}\left(W_{b}\right), u_{1}^{\gamma}\right)\right)$ with probability at least $\epsilon$.

Claim 3: Suppose that in a real attack game $\mathcal{A}$ is given the public key $\left[g, u_{1}\right]$ and $\mathcal{A}$ asks to be challenged on words $W_{0}$ and $W_{1}$. In response, $\mathcal{A}$ is given a challenge $C=\left[g^{r}, J\right]$. Then, in the real attack game $\mathcal{A}$ issues an $H_{2}$ query for either $H_{2}\left(e\left(H_{1}\left(W_{0}\right), u_{1}^{r}\right)\right)$ or $H_{2}\left(e\left(H_{1}\left(W_{1}\right), u_{1}^{r}\right)\right)$ with probability at least $2 \epsilon$.

Proof. Let $\mathcal{E}_{3}$ be the event that in the real attack $\mathcal{A}$ does not issue a query for either one of $H_{2}\left(e\left(H_{1}\left(W_{0}\right), u_{1}^{r}\right)\right)$ and $H_{2}\left(e\left(H_{1}\left(W_{1}\right), u_{1}^{r}\right)\right)$. Then, when $\mathcal{E}_{3}$ occurs we know that the bit $b \in\{0,1\}$ indicating whether $C$ is a PEKS of $W_{0}$ or $W_{1}$ is independent of $\mathcal{A}$ 's view. Therefore, $\mathcal{A}$ 's output $b^{\prime}$ will satisfy $b=b^{\prime}$ with probability at most $\frac{1}{2}$. By definition of $\mathcal{A}$, we know that in the real attack $\left|\operatorname{Pr}\left[b=b^{\prime}\right]-1 / 2\right| \geq \epsilon$. We show that these two facts imply that $\operatorname{Pr}\left[\neg \mathcal{E}_{3}\right] \geq 2 \epsilon$. To do so, we first derive simple upper and lower bounds on $\operatorname{Pr}\left[b=b^{\prime}\right]$ :

$$
\begin{aligned}
& \operatorname{Pr}\left[b=b^{\prime}\right]=\operatorname{Pr}\left[b=b^{\prime} \mid \mathcal{E}_{3}\right] \operatorname{Pr}\left[\mathcal{E}_{3}\right]+\operatorname{Pr}\left[b=b^{\prime} \mid \neg \mathcal{E}_{3}\right] \operatorname{Pr}\left[\neg \mathcal{E}_{3}\right] \\
& \leq \operatorname{Pr}\left[b=b^{\prime} \mid \mathcal{E}_{3}\right] \operatorname{Pr}\left[\mathcal{E}_{3}\right]+\operatorname{Pr}\left[\neg \mathcal{E}_{3}\right] \\
&=\frac{1}{2} \operatorname{Pr}\left[\mathcal{E}_{3}\right]+\operatorname{Pr}\left[\neg \mathcal{E}_{3}\right] \\
&=\frac{1}{2}+\frac{1}{2} \operatorname{Pr}\left[\neg \mathcal{E}_{3}\right], \\
& \operatorname{Pr}\left[b=b^{\prime}\right] \geq \operatorname{Pr}\left[b=b^{\prime} \mid \mathcal{E}_{3}\right] \operatorname{Pr}\left[\mathcal{E}_{3}\right]=\frac{1}{2} \operatorname{Pr}\left[\mathcal{E}_{3}\right]=\frac{1}{2}-\frac{1}{2} \operatorname{Pr}\left[\neg \mathcal{E}_{3}\right] .
\end{aligned}
$$

It follows that $\epsilon \leq\left|\operatorname{Pr}\left[b=b^{\prime}\right]-1 / 2\right| \leq \frac{1}{2} \operatorname{Pr}\left[\neg \mathcal{E}_{3}\right]$. Therefore, in the real attack, $\operatorname{Pr}\left[\neg \mathcal{E}_{3}\right] \geq 2 \epsilon$ as required.

Now, assuming $\mathcal{B}$ does not abort, we know that $\mathcal{B}$ simulates a real attack game perfectly up to the moment when $\mathcal{A}$ issues a query for either $H_{2}\left(e\left(H_{1}\left(W_{0}\right), u_{1}^{\gamma}\right)\right)$ or $H_{2}\left(e\left(H_{1}\left(W_{1}\right), u_{1}^{\gamma}\right)\right)$. Therefore, by Claim 3 , by the end of the simulation $\mathcal{A}$ will have issued a query for either $H_{2}\left(e\left(H_{1}\left(W_{0}\right), u_{1}^{\gamma}\right)\right)$ or $H_{2}\left(e\left(H_{1}\left(W_{1}\right), u_{1}^{\gamma}\right)\right)$ with probability at least $2 \epsilon$. It follows that $\mathcal{A}$ issues a query for $H_{2}\left(e\left(H_{1}\left(W_{b}\right), u_{1}^{\gamma}\right)\right)$ with probability at least $\epsilon$. Consequently, the value $e\left(H_{1}\left(W_{b}\right), u_{1}^{\gamma}\right)=e\left(g^{\beta+a_{b}}, g\right)^{\alpha \gamma}$ will appear on the left hand side of some pair in the $\mathrm{H}_{2}$-list. Algorithm $\mathcal{B}$ will 
choose the correct pair with probability at least $1 / q_{H_{2}}$ and therefore, assuming $\mathcal{B}$ does not abort during the simulation, it will produce the correct answer with probability at least $\epsilon / q_{\mathrm{H}_{2}}$. Since $\mathcal{B}$ does not abort with probability at least $1 /\left(e q_{T}\right)$ we see that $\mathcal{B}$ 's success probability overall is at least $\epsilon /\left(e q_{T} q_{H_{2}}\right)$ as required.

\subsection{A Limited Construction Using Any Trapdoor Permutation}

Our second PEKS construction is based on general trapdoor permutations, assuming that the total number of keywords that the user wishes to search for is bounded by some polynomial function in the security parameter. (As a first step in our construction, we will make an even stronger assumption that the total number of words $\Sigma \subset\{0,1\}^{*}$ in the dictionary is also bounded by a polynomial function, we will later show how to remove this additional assumption.) We will also need a family of semantically-secure encryptions where given a ciphertext it is computationally hard to say which public-key this ciphertext is associated with. This notion was formalized by Bellare et al. [1. We say that a public-key system that has this property is source-indistinguishable. More precisely, source-indistinguishability for an encryption scheme $(G, E, D)$ is defined using the following game between a challenger and an attacker $\mathcal{A}$ (here $G$ is the key generation algorithm, and $E / D$ are encryption/decryption algorithms). The security parameter $s$ is given to both players.

Source Indistinguishability security game:

1. The challenger runs algorithm $G(s)$ two times to generate two public/private key pairs $\left(P K_{0}\right.$, Priv 0$)$ and $\left(P K_{1}\right.$, Priv $\left._{1}\right)$.

2. The challenger picks a random $M \in\{0,1\}^{s}$ and a random $b \in\{0,1\}$ and computes an encryption $C=P K_{b}(M)$. The challenger gives $(M, C)$ to the attacker.

3. The attacker outputs $b^{\prime}$ and wins the game if $b=b^{\prime}$.

In other words, the attacker wins if he correctly guesses whether he was given the encryption of $M$ under $P K_{0}$ or under $P K_{1}$. We define $\mathcal{A}$ 's advantage in winning the game as:

$$
\operatorname{AdvSI}_{\mathcal{A}}(s)=\left|\operatorname{Pr}\left[b=b^{\prime}\right]-\frac{1}{2}\right|
$$

Definition 3. We say that a public-key encryption scheme is source indistinguishable if for any polynomial time attacker $\mathcal{A}$ we have that $\operatorname{AdvSI}_{\mathcal{A}}(s)$ is a negligible function.

We note that Bellare et al. [1] define a stronger notion of source indistinguishability than the one above by allowing the adversary to choose the challenge message $M$. For our purposes, giving the adversary an encryption of a random message is sufficient.

It is easy to check that source indistinguishability can be attained from any trapdoor permutation family, where for a given security parameter all permutations in the family are defined over the same domain. Such a family can be 
constructed from any family of trapdoor permutations as described in 11. Then to encrypt a bit $b$ we pick a random $x$, and output $[f(x), G L(x) \oplus b]$ where GL is the Goldreich-Levin hard-core bit [19]. We therefore obtain the following lemma:

Lemma 2. Given any trapdoor permutation family we can construct a semantically secure source indistinguishable encryption scheme.

We note that source indistinguishability is an orthogonal property to semantic security. One can build a semantically secure system that is not source indistinguishable (by embedding the public key in every ciphertext). Conversely, one can build a source indistinguishable system that is not semantically secure (by embedding the plaintext in every ciphertext).

A simple PEKS from trapdoor permutations. When the keyword family $\Sigma$ is of polynomial size (in the security parameter) it is easy to construct searchable encryption from any source-indistinguishable public-key system $(G, E, D)$. We let $s$ be the security parameter for the scheme.

- KeyGen: For each $W \in \Sigma$ run $G(s)$ to generate a new public/private key pair $P K_{W} / \operatorname{Priv}_{W}$ for the source-indistinguishable encryption scheme. The PEKS public key is

$A_{\text {pub }}=\left\{P K_{W} \mid W \in \Sigma\right\}$. The private key is $A_{\text {priv }}=\left\{\operatorname{Priv}_{W} \mid W \in \Sigma\right\}$.

- $\operatorname{PEKS}\left(A_{\text {pub }}, W\right)$ : Pick a random $M \in\{0,1\}^{s}$ and output $\operatorname{PEKS}\left(A_{\text {pub }}, W\right)=$ $\left(M, E\left[P K_{W}, M\right]\right)$, i.e. encrypt $M$ using the public key $P K_{W}$.

- Trapdoor $\left(A_{\text {priv }}, W\right)$ : The trapdoor for word $W$ is simply $T_{W}=\operatorname{Priv}_{W}$.

- Test $\left(A_{p u b}, S, T_{W}\right)$ : Test if the decryption $D\left[T_{W}, S\right]=0^{s}$. Output 'yes' if so and 'no' otherwise.

Note that the dictionary must be of polynomial size (in $s$ ) so that the public and private keys are of polynomial size (in $s$ ).

This construction gives a semantically secure PEKS as stated in the following simple theorem. Semantically secure PEKS is defined as in Definition 2 except that the adversary is not allowed to make chosen keyword queries.

Theorem 2. The PEKS scheme above is semantically secure assuming the underlying public key encryption scheme $(G, E, D)$ is source-indistinguishable.

Proof sketch: Let $\Sigma=\left\{W_{1}, \ldots, W_{k}\right\}$ be the keyword dictionary. Suppose we have a PEKS attacker $\mathcal{A}$ for which $\operatorname{Adv}_{\mathcal{A}}(s)>\epsilon(s)$. We build an attacker $\mathcal{B}$ that breaks the source indistinguishability of $(G, E, D)$ where $\operatorname{AdvSI}_{\mathcal{B}}(s)>\epsilon(s) / k^{2}$.

The reduction is immediate: $\mathcal{B}$ is given two public keys $P K_{0}, P K_{1}$ and a pair $(M, C)$ where $M$ is random in $\{0,1\}^{s}$ and $C=P K_{b}(M)$ for $b \in\{0,1\}$. Algorithm $\mathcal{B}$ generates $k-2$ additional public/private keys using $G(s)$. It creates $A_{\text {pub }}$ as a list of all $k$ public keys with $P K_{0}, P K_{1}$ embedded in a random location in the list. Let $W_{i}, W_{j}$ be the words associated with the public keys $P K_{0}, P K_{1}$. $\mathcal{B}$ sends $A_{\text {pub }}$ to $\mathcal{A}$ who then responds with two words $W_{k}, W_{\ell} \in \Sigma$ on which $\mathcal{A}$ wishes to be challenged. If $\{i, j\} \neq\{k, \ell\}$ algorithm $\mathcal{B}$ reports failure and aborts. Otherwise, $\mathcal{B}$ sends the challenge $(M, C)$ to $\mathcal{A}$ who then responds with a $b^{\prime} \in$ 
$\{0,1\}$. Algorithm $\mathcal{B}$ outputs $b^{\prime}$ as its response to the source indistinguishability challenge. We have that $b=b^{\prime}$ if algorithm $\mathcal{B}$ did not abort and $\mathcal{A}$ 's response was correct. This happens with probability at least $\frac{1}{2}+\epsilon / k^{2}$. Hence, $\operatorname{AdvSI}_{\mathcal{B}}(s)>$ $\epsilon(s) / k^{2}$ as required.

We note that this PEKS can be viewed as derived from an IBE system with a limited number of identities. For each identity there is a pre-specified public key. Such an IBE system is implied in the work of Dodis et al. 13. They propose reducing the size of the public-key using cover-free set systems. We apply the same idea below to reduce the size of the public key in the PEKS above.

Reducing the public key size. The drawback of the above scheme is that the public key length grows linearly with the total dictionary size. If we have an upper-bound on the total number of keyword trapdoors that the user will release to the email gateway (though we do not need to know these keywords a-priori) we can do much better using cover-free families [15] and can allow keyword dictionary to be of exponential size. Since typically a user will only allow a third party (such as e-mail server) to search for a limited number of keywords so that assuming an upper bound on the number of released trapdoors is within reason. We begin by recalling the definition of cover-free families.

Definition 4. Cover-free families. Let $d, t, k$ be positive integers, let $G$ be a ground set of size $d$, and let $F=\left\{S_{1}, \ldots, S_{k}\right\}$ be a family of subsets of $G$. We say that subset $S_{j}$ does not cover $S_{i}$ if it holds that $S_{i} \not \subseteq S_{j}$. We say that family $F$ is $t$-cover free over $G$ if each subset in $F$ is not covered by the union of $t$ subsets in F. Moreover, we say that a family of subsets is q-uniform if all subsets in the family have size $q$.

We will use the following fact from [14].

Lemma 3. 14 There exists a deterministic algorithm that, for any fixed $t, k$, constructs a $q$-uniform $t$-cover free family $F$ over a ground set of size $d$, for $q=\lceil d / 4 t\rceil$ and $d \leq 16 t^{2}(1+\log (k / 2) / \log 3)$.

The PEKS. Given the previous PEKS construction as a starting point, we can significantly reduce the size of public file $A_{p u b}$ by allowing user to re-use individual public keys for different keywords. We associate to each keyword a subset of public keys chosen from a cover free family. Let $k$ be the size of the dictionary $\Sigma=\left\{W_{1}, \ldots, W_{k}\right\}$ and let $t$ be an upper bound on the number of keyword trapdoors released to the mail gateway by user Alice. Let $d, q$ satisfy the bounds of Lemma 3 The PEKS $(d, t, k, q)$ construction is as follows:

- KeyGen: For $i=1, \ldots, d$ run algorithm $G(s)$ to generate a new public/private key pair $P K_{i} /$ Priv $_{i}$ for the source-indistinguishable encryption scheme. The PEKS public key is $A_{\text {pub }}=\left\{P K_{1}, \ldots, P K_{d}\right\}$. The private key is $A_{\text {priv }}=$ $\left\{\right.$ Priv $_{1}, \ldots$, Priv $\left._{d}\right\}$. We will be using a $q$-uniform $t$-cover free family of subsets $F=\left\{S_{1}, \ldots, S_{k}\right\}$ of $\left\{P K_{1}, \ldots, P K_{d}\right\}$. Hence, each $S_{i}$ is a subset of public keys. 
- PEKS $\left(A_{\text {pub }}, W_{i}\right)$ : Let $S_{i} \in F$ be the subset associated with the word $W_{i} \in \Sigma$. Let $S_{i}=\left\{P K^{(1)}, \ldots, P K^{(q)}\right\}$. Pick random messages $M_{1}, \ldots, M_{q} \in\{0,1\}^{s}$ and let $M=M_{1} \oplus \cdots \oplus M_{q}$. Output the tuple:

$$
\operatorname{PEKS}\left(A_{p u b}, W_{i}\right)=\left(M, E\left[P K^{(1)}, M_{1}\right], \ldots, E\left[P K^{(q)}, M_{q}\right]\right)
$$

- Trapdoor $\left(A_{\text {priv }}, W_{i}\right)$ : Let $S_{i} \in F$ be the subset associated with word $W_{i} \in \Sigma$. The trapdoor for word $W_{i}$ is simply the set of private keys that correspond to the public keys in the set $S_{i}$.

- Test $\left(A_{\text {pub }}, R, T_{W}\right)$ :

Let $T_{W}=\left\{\operatorname{Priv}^{(1)}, \ldots, \operatorname{Priv}^{(q)}\right\}$ and let $R=\left(M, C_{1}, \ldots, C_{q}\right)$ be a PEKS. For $i=1, \ldots, q$ decrypt each $C_{i}$ using private key $\operatorname{Priv}^{(i)}$ to obtain $M_{i}$. Output 'yes' if $M=M_{1} \oplus \cdots \oplus M_{q}$, and output 'no' otherwise.

The size of the public key file $A_{\text {pub }}$ is much smaller now: logarithmic in the size of the dictionary. The downside is that Alice can only release $t$ keywords to the email gateway. Once $t$ trapdoors are released privacy is no longer guaranteed. Also, notice that the size of the PEKS is larger now (logarithmic in the dictionary size and linear in $t$ ). The following corollary of Theorem 2 shows that the resulting PEKS is secure.

Corollary 1. Let $d, t, k, q$ satisfy the bounds of Lemma 圆. The PEKS $(d, t, k, q)$ scheme above is semantically secure under a chosen keyword attack assuming the underlying public key encryption scheme $(G, E, D)$ is source-indistinguishable and semantically secure, and that the adversary makes no more than $t$ trapdoors queries.

Proof sketch: Let $\Sigma=\left\{W_{1}, \ldots, W_{k}\right\}$ be the keyword dictionary. Suppose we have a PEKS attacker $\mathcal{A}$ for which $\operatorname{Adv}_{\mathcal{A}}(s)>\epsilon(s)$. We build an attacker $\mathcal{B}$ that breaks the source indistinguishability of $(G, E, D)$.

Algorithm $\mathcal{B}$ is given two public keys $P K_{0}, P K_{1}$ and a pair $(M, C)$ where $M$ is random in $\{0,1\}^{s}$ and $C=P K_{b}(M)$ for $b \in\{0,1\}$. Its goal is to output a guess for $b$ which it does by interacting with $\mathcal{A}$. Algorithm $\mathcal{B}$ generates $d-2$ additional public/private keys using $G(s)$. It creates $A_{\text {pub }}$ as a list of all $d$ public keys with $P K_{0}, P K_{1}$ embedded in a random location in the list. Let $W_{i}, W_{j}$ be the words associated with the public keys $P K_{0}, P K_{1}$.

$\mathcal{B}$ sends $A_{\text {pub }}$ to $\mathcal{A}$. Algorithm $\mathcal{A}$ issues up to $t$ trapdoor queries. $\mathcal{B}$ responds to a trapdoor query for $W \in \Sigma$ as follows: let $S \in F$ be the subset corresponding to the word $W$. If $P K_{0} \in S$ or $P K_{1} \in S$ algorithm $\mathcal{B}$ reports failure and aborts. Otherwise, $\mathcal{B}$ gives $\mathcal{A}$ the set of private keys $\left\{\operatorname{Priv}_{i} \mid i \in S\right\}$.

At some point, Algorithm $\mathcal{A}$ outputs two words $W_{0}^{\prime}, W_{1}^{\prime} \in \Sigma$ on which it wishes to be challenged. Let $S_{0}^{\prime}, S_{1}^{\prime} \in F$ be the subsets corresponding to $W_{0}^{\prime}, W_{1}^{\prime}$ respectively. Let $\mathcal{E}$ be the event that $P K_{0} \in S_{0}^{\prime}$ and $P K_{1} \in S_{1}^{\prime}$. If event $\mathcal{E}$ did not happen then $\mathcal{B}$ reports failure and aborts.

We now know that $P K_{0} \in S_{0}^{\prime}$ and $P K_{1} \in S_{1}^{\prime}$. For $j=0,1$ let $S_{j}^{\prime}=$ $\left\{P K_{j}^{(1)}, \ldots, P K_{j}^{(q)}\right\}$. We arrange things so that $P K_{0}=P K_{0}^{(c)}$ and $P K_{1}=$ 
$P K_{1}^{(c)}$ for some random $1 \leq c \leq q$. Next, $\mathcal{B}$ picks random $M_{1}, \ldots, M_{c-1}$, $M_{c+1}, \ldots, M_{q} \in\{0,1\}^{s}$ and sets $M_{c}=M$. Let $M^{\prime}=M_{1} \oplus \cdots \oplus M_{q}$. Algorithm $\mathcal{B}$ defines the following hybrid tuple:

$$
\begin{aligned}
& R=\left(M^{\prime}, E\left[P K_{0}^{(1)}, M_{1}\right]\right. \ldots, E\left[P K_{0}^{(c-1)}, M_{c-1}\right], C, \\
&\left.E\left[P K_{1}^{(c+1)}, M_{c+1}\right], \ldots, E\left[P K_{1}^{(q)}, M_{q}\right]\right)
\end{aligned}
$$

It gives $R$ as the challenge PEKS to algorithm $\mathcal{A}$. Algorithm $\mathcal{A}$ eventually responds with some $b^{\prime} \in\{0,1\}$ indicating whether $R$ is $\operatorname{PEKS}\left(A_{p u b}, W_{0}^{\prime}\right)$ or $\operatorname{PEKS}\left(A_{\text {pub }}, W_{1}^{\prime}\right)$. Algorithm $\mathcal{B}$ outputs $b^{\prime}$ as its guess for $b$. One can show using a standard hybrid argument that if $\mathcal{B}$ does not abort then $\left|\operatorname{Pr}\left[b=b^{\prime}\right]-\frac{1}{2}\right|>\epsilon / q^{2}$. The probability that $\mathcal{B}$ does not abort at a result of a trapdoor query is at least $1-(t q / d)$. The probability that $\mathcal{B}$ does not abort as a result of the choice of words $W_{0}^{\prime}, W_{1}^{\prime}$ is at least $(q / d)^{2}$. Hence, $\mathcal{B}$ does not abort with probability at least $1 / \operatorname{poly}(t, q, d)$. Repeatedly running $\mathcal{B}$ until it does not abort shows that we can get advantage $\epsilon / q^{2}$ in breaking the source indistinguishability of $(G, E, D)$ in expected polynomial time in the running time of $\mathcal{A}$.

\section{Construction Using Jacobi Symbols}

Given the relation between Identity Based Encryption and PEKS it is tempting to construct a PEKS from an IBE system due to Cocks [3]. The security of Cocks' IBE system is based on the difficulty of distinguishing quadratic residues from non-residues modulo $N=p q$ where $p=q=3(\bmod 4)$.

Unfortunately, Galbraith [11] shows that the Cocks system as described in [3] is not public-key private in the sense of Bellare et al. [1]. Therefore it appears that the Cocks system cannot be directly used to construct a PEKS. It provides a good example that constructing a PEKS is a harder problem than constructing an IBE.

\section{Conclusions}

We defined the concept of a public key encryption with keyword search (PEKS) and gave two constructions. Constructing a PEKS is related to Identity Based Encryption (IBE), though PEKS seems to be harder to construct. We showed that PEKS implies Identity Based Encryption, but the converse is currently an open problem. Our constructions for PEKS are based on recent IBE constructions. We are able to prove security by exploiting extra properties of these schemes.

\section{Acknowledgments}

We thank Glenn Durfee for suggesting the use of $H_{2}$ in the construction of Section 3.1. We thank Yevgeniy Dodis, David Molnar, and Steven Galbraith for helpful comments on this work. 


\section{References}

1. M. Bellare, A. Boldyreva, A. Desai, and D. Pointcheval "Key-Privacy in PublicKey Encryption," in Advances in Cryptology - Asiacrypt 2001 Proceedings, LNCS Vol. 2248, Springer-Verlag, 2001.

2. D. Boneh and M. Franklin, Identity-based Encryption from the Weil Pairing, SIAM J. of Computing, Vol. 32, No. 3, pp. 586-615, 2003, Extended abstract in Crypto 2001.

3. C. Cocks, An identity based encryption scheme based on quadratic residues, Eighth IMA International Conference on Cryptography and Coding, Dec. 2001, Royal Agricultural College, Cirencester, UK.

4. C. Cachin, S. Micali, M. Stadler Computationally Private Information Retrieval with Polylogarithmic Communication Eurcrypt 1999.

5. B. Chor, O. Goldreich, E. Kushilevitz and M. Sudan, Private Information Retrieval, in FOCS 95 (also Journal of ACM).

6. J. Coron, "On the exact security of Full-Domain-Hash", in Advances in Cryptology - Crypto 2000, Lecture Notes in Computer Science, Vol. 1880, Springer-Verlag, pp. 229-235, 2000.

7. D. Dolev, C. Dwork, and M. Naor, "Non-Malleable Cryptography," in SIAM Journal on Computing, 2000. Early version in proceedings of STOC '91.

8. G. Di Crescenzo, Y. Ishai, and R. Ostrovsky. Universal service-providers for database private information retrieval. In Proc. of the 17th Annu. ACM Symp. on Principles of Distributed Computing, pages 91-100, 1998.

9. G. Di Crescenzo, T. Malkin, and R. Ostrovsky. Single-database private information retrieval implies oblivious transfer. In Advances in Cryptology - EUROCRYPT 2000, 2000.

10. A. Iliev, S. Smith Privacy-enhanced credential services. Second annual PKI workshop. (see also Darthmoth Technical Report TR-2003-442; http://www.cs.dartmouth.edu/ sws/papers/ilsm03.pdf.

11. S. Galbraith, private communications.

12. Y. Desmedt, "Computer security by redefining what a computer is," in Proceedings New Security Paradigms II Workshop, pp. 160-166, 1992.

13. Y. Dodis, J. Katz, S. Xu, and M. Yung. "Key-insulated public key cryptosystems," in Advances in Cryptology - Eurocrypt 2002, LNCS, Springer-Verlag, pp. 65-82, 2002.

14. D. Z. Du and F. K. Hwang, Combinatorial Group Testing and its Applications, World Scientific, Singapore, 1993.

15. P. Erdos, P. Frankl and Z. Furedi, Families of finite sets in which no set is covered by the union of $r$ others, in Israeli Journal of Mathematics, 51: 79-89, 1985.

16. E. Goh, "Building Secure Indexes for Searching Efficiently on Encrypted Compressed Data," http://eprint.iacr.org/2003/216/

17. O. Goldreich and R. Ostrovsky. Software protection and simulation by oblivious RAMs. JACM, 1996.

18. Goldreich, O., S. Goldwasser, and S. Micali, "How To Construct Random Functions," Journal of the Association for Computing Machinery, Vol. 33, No. 4 (October 1986), 792-807.

19. S. Goldwasser and S. Micali, Probabilistic Encryption, in Journal of Computer and System Sciences. vol. 28 (1984), n. 2, pp. 270-299.

20. Y. Gertner, Y. Ishai, E. Kushilevitz, and T. Malkin Protecting data privacy in private information retrieval schemes. In Proc. of the 30th Annual ACM Symposium on the Theory of Computing, pp. 151-160, 1998. 
21. A. Joux, "The Weil and Tate Pairings as Building Blocks for Public Key Cryptosystems", in Proc. Fifth Algorithmic Number Theory Symposium, Lecture Notes in Computer Science, Springer-Verlag, 2002.

22. E. Kushilevitz and R. Ostrovsky, Replication is not needed: Single Database, Computationally-Private Information Retrieval, in FOCS 97.

23. E. Kushilevitz and R. Ostrovsky. One-way Trapdoor Permutations are Sufficient for Non-Trivial Single-Database Computationally-Private Information Retrieval. In Proc. of EUROCRYPT '00, 2000.

24. P. Maniatis, M. Roussopoulos, E. Swierk, K. Lai, G. Appenzeller, X. Zhao, and M. Baker, The Mobile People Architecture. ACM Mobile Computing and Communications Review (MC2R), Volume 3, Number 3, July 1999.

25. M. Naor and B. Pinkas. Oblivious transfer and polynomial evaluation. In Proc. of the 31th Annu. ACM Symp. on the Theory of Computing, pages 245-254, 1999.

26. R. Ostrovsky. Software protection and simulation on oblivious RAMs. MIT Ph.D. Thesis, 1992. Preliminary version in Proc. 22nd Annual ACM Symp. Theory Comp., 1990.

27. W. Ogata and K. Kurosawa, "Oblivious keyword search," to appear in J. of Complexity.

28. D. Song, D. Wagner, and A. Perrig, Practical Techniques for Searches on Encrypted Data, in Proc. of the 2000 IEEE symposium on Security and Privacy (S\&P 2000).

29. A. Shamir, Identity-based Cryptosystems and Signature Schemes, in CRYPTO 84.

30. B. Waters, D. Balfanz, G. Durfee, D. Smetters, "Building an encrypted and searchable audit log", to appear in NDSS '04. 